\title{
SmartRES - Uma plataforma IoT para Monitoramento Inteligente em Saúde e sua Aplicação no Contexto da COVID-19
}

\author{
Vinícius N. Barbosa ${ }^{1}$, Helen Abdala R. Ferreira ${ }^{1}$, Leonardo Gomes ${ }^{1}$, Fábio Gomes ${ }^{1}$, \\ Ivana Barreto $^{2}$, Odorico Monteiro ${ }^{2}$, Mauro Oliveira ${ }^{1}$ \\ ${ }^{1}$ Instituto Federal de Educação, Ciência e Tecnologia do Ceará (IFCE) \\ \{viniciusnb14, helenabdala14,prof.fabiojose, mauroboliveira\}@gmail.com \\ leonardo.gomes@ppgcc.ifce.edu.br \\ ivana.barretoefiocruz.br \\ odorico0811@gmail.com
}

\begin{abstract}
The Brazilian Ministry of Health launched the concept of Minimum Data Set, a tool that will integrate the registration of patient data in a single layout. On the other hand, private health plans have adopted different approaches in the implementation of Electronic Health Records (EHR), using different standards of semantic interoperability. In this context, SmartRES proposes an intelligent health management platform, which will allow the interoperability of different public and private EHR. With SmartRES, a user's clinical information can be accessed anytime and from any location. SmartRES intelligent services will be able to alert the user in the prevention and prediction of clinical problems by crossing their history with real-time data. Finally, the paper presents the use of the SmartRES platform in the new context of COVID-19.
\end{abstract}

Resumo. O Ministério da Saúde no Brasil lançou o conceito de Conjunto Mínimo de Dados $(C M D)^{1}$, ferramenta que integrará o registro dos dados do paciente em um layout único. Por outro lado, os planos de saúde privados têm adotado diferentes abordagens na implantação dos Registros Eletrônicos de Saúde (RES), utilizando diferentes padrões de interoperabilidade semântica. Nesse contexto, o SmartRES propõe uma plataforma inteligente de gestão em saúde, que permitirá a interoperabilidade de diferentes RES públicos e privados. Com o SmartRES as informações clínicas de um usuário poderão ser acessadas a qualquer hora e lugar. Serviços inteligentes do SmartRES poderão alertar o usuário na prevenção e predição de problemas clínicos a partir do cruzamento de seu histórico, com dados em tempo real. Finalmente, o trabalho apresenta o uso da plataforma SmartRES no novo contexto da COVID-19.

\section{Introdução}

Os Sistemas de Informação em Saúde (SIS) contribuem para a eficácia e melhoria do atendimento em saúde, em especial ao autocuidado. Dessa forma, é correto afirmar que

\footnotetext{
${ }^{1}$ https://conjuntominimo.saude.gov.br//
} 
um SIS gerencia informações provenientes de profissionais de saúde, a fim de aprimorar suas atividades. Ademais, o SIS também facilita a comunicação, bem como integrar informações e auxiliar na tomada de decisão, fornecendo apoio financeiro e administrativo [Safran and Perreault 2001].

Atualmente, as entidades de saúde têm buscado cada vez mais modelos de saúde que possuem maior integração. Para tanto, soluções como o RES e o Prontuário Eletrônico do Paciente (PEP) foram concebidos. O RES pode ser definido, lato senso, como um repositório de informações de saúde do paciente que permite o compartilhamento destes dados entre sistemas e unidades públicas ou privadas. Estruturalmente, o RES é mais rico em informação do que o PEP, que contém dados locais sobre a saúde de um paciente, armazenados e sob a guarda de uma determinada organização de saúde. Um mesmo paciente pode ter informações clínicas em diversos PEPs, por exemplo: informações no PEP de um hospital ou de um consultório médico ou, ainda, em um pronto atendimento. Em geral, estes PEPs não são compatíveis dos pontos de vista sintático, semântico e computacional. O objetivo do RES é, portanto, prover a interoperabilidade das informações dos diversos PEPs, ou seja, compartilhar as informações clínicas relevantes dos diversos PEPs, disponibilizando-as em uma visão integrada, o que resulta na economia de recursos e de tempo, facilitando o processo de tomada de decisão em todos os níveis da gestão de saúde.

Recentemente, o Ministério da Saúde deu um passo importante para informatização do Sistema Único de Saúde (SUS) no aprimoramento do registro de dados de serviços, reduzindo custos e tempo de alimentação por parte dos gestores de saúde. Trata-se do CMD[Atenção à Saúde 2015], ferramenta que integrará o registro dos dados do paciente em todos os estabelecimentos públicos e privados de saúde em território nacional com um layout único, evitando a distribuição da mesma informação em sistemas diferentes, provocando economia financeira e de tempo relacionado à manutenção de cada sistema.

Integrar sistemas já existentes no SUS não tem sido uma tarefa fácil para o DATASUS $^{2}$ (Departamento de Informática do SUS). Além disso, os Planos de Saúde privados têm adotado diferentes abordagens na implantação do RES, utilizando outros padrões de interoperabilidade, como o Fast Healthcare Interoperability Resources (FHIR) que possuem diferentes arquiteturas de interoperabilidade. Assim, imaginar um RES interoperável no país que contemple ambientes públicos e privados é uma tarefa nada trivial.

Ao se tentar resolver problemas relacionados à interoperabilidade e integração em sistemas de saúde, vale ressaltar a utilização de tecnologias atuais que auxiliam na melhoria do processo. Internet das Coisas (do inglês, Internet of Things - IoT) tem mostrado cada vez mais potencial para assistência à saúde, com aplicações que podem ser constituídas em parte ou totalmente por dispositivos inteligentes [Costa 2018].

Este trabalho apresenta o SmartRES, um sistema de monitoramento para apoio ao usuário (pacientes ou não) em situações de urgência e emergência. Um cenário hipotético é apresentado onde as informações clínicas do usuário podem ser acessadas a qualquer hora e lugar. Serviços inteligentes agregados poderão prevenir o usuário a partir do cruzamento de seu histórico (RES) com dados em tempo real (sensores de sinais vitais).

\footnotetext{
${ }^{2}$ https://datasus.saude.gov.br
} 
O SmartRES faz uso de dois importantes componentes:

- O MARCIA [Gomes et al. 2018], uma metodologia para o desenvolvimento de sistemas interoperáveis, utilizando a estrutura EHRServer do padrão OpenEHR.

- O GIRLS [Gomes et al. 2019], um gateway de baixo custo para interoperabilidade de RES que usa ambos os padrões FHIR e OpenEHR.

\section{Trabalhos Relacionados}

O desenvolvimento e utilização de soluções para tornar os sistemas de saúde interoperáveis está cada vez mais popularizado, resultado de diversos trabalhos que obtiveram êxito em sua implementação.

Em [Paz et al. 2013], os autores propõem uma arquitetura aliada à Computação Ubíqua, nomeada de MobiDoctor, voltada para dispositivos móveis que possibilita a consulta ao RES de informações médicas do paciente em ambientes hospitalares. O trabalho utiliza o padrão Health Level Seven ${ }^{3}$ de troca de mensagens voltado para dados clínicos e administrativos, capaz de gerenciar e compartilhar informações relacionadas aos pacientes com aplicativos.

No trabalho de [Costa et al. 2011] os autores focam na transformação de arquétipos para interoperar semanticamente os padrões de RES, baseado na arquitetura de modelo duplo. A metodologia proposta visa possibilitar a troca de dados clínicos entre sistemas de diferentes padrões e foi aplicada à ISO 13606 e ao Open Electronic Health Records (OpenEHR). Para isto, os autores combinam modelos de referências, arquétipos e ontologias com o suporte de técnicas de engenharia orientada a modelos.

Na dissertação de [Braga et al. 2014], a autora elucida a importância de sistemas interoperáveis e enaltece a utilização de ontologias para lidar com a falta de interoperabilidade entre sistemas primários de saúde. Nele, a autora aborda as técnicas de ontologias formais e informais, a fim de suprir certas carências do padrão OpenEHR para tal tarefa.

\section{SmartRES}

A Figura 1 apresenta a arquitetura funcional do SmartRES, o sistema proposto neste trabalho, capaz de monitorar o usuário em tempo real com ações de prevenção e alertas que são processados e efetivados a partir de variações observadas nos dados coletados em tempo real. Uma vez coletados, esses dados são enviados para a camada de predição, o CloudRES que inicia uma análise dos dados recebidos (Módulos Sensores e Aquisição de Dados) e avalia a relevância do alerta emitido, bem como o nível do risco (Módulo de Nível de Risco). Caso o alerta seja relevante, ações para auxiliar o usuário são iniciadas.

\subsection{Módulo Sensores}

O usuário do SmartRES será monitorado por meio de sensores de sinais vitais como (Módulo Sensores): pressão arterial, SPO2 (saturação do oxigênio no sangue), temperatura, entre outros. Esses dados serão coletados em tempo real por uma pulseira e enviados para a aplicação MobileRES (Módulo Serviços), para serem visualizados pelo usuário em um dashboard. Caso ocorra uma variação nos resultados (gerador de alerta), os dados são enviados para o componente CloudRES para análise da relevância e o nível do risco (Módulo Nivel de Risco).

\footnotetext{
${ }^{3}$ http://www.hl 7.com.br/
} 
Figura 1. Arquitetura Funcional do SmartRES.



\subsection{Módulo Aquisição dos Dados}

Este Módulo é constituído por três componentes: RES, MARCIA e GIRLS.

O RES é um repositório de informações processáveis sobre o cuidado em saúde do indivíduo, armazenadas e transmitidas de forma segura e acessível por múltiplos usuários autorizados. Seu principal objetivo e oferecer apoio a cuidados de saúde de qualidade, eficazes, eficientes, efetivos, seguros e integrados, ao longo de toda a vida do paciente [Atenção à Saúde 2015]. Assim, o componente RES no SmartRES funciona como um sistema completo para registro de todas as etapas de atendimento de pacientes, que permite a criação e arquivo do histórico clínico. Quando utilizado de forma correta, um RES pode diminuir consideravelmente a incidência de erros médicos, sobretudo nos casos em que os pacientes migram de um profissional para outro.

O MARCIA é um sistema de gerenciamento aplicado em registros clínicos. Ele propõe uma metodologia para o desenvolvimento de sistemas flexíveis, utilizando a estrutura EHRServer da norma OpenEHR. Como estudo de caso, essa metodologia foi aplicada em Aracati/CE desde março/2017, no contexto da doença de Chikungunya. A metodologia é suportada por um sistema que implementa um conjunto de arquétipos OpenEHR representando o tratamento clínico da Chikungunya. O sistema foi testado em uma Unidade Básica de saúde. Os arquétipos e os modelos MARCIA foram disponibilizados para o Clinical Knowledge Manager (CKM), o maior repositório online de arquétipos na Web [Gomes et al. 2018].

O GIRLS é um gateway de baixo custo para interoperabilidade dos padrões FHIR e OpenEHR, fornecendo uma solução viável para troca de dados entre sistemas. Além 
disso, ele fornece a interoperabilidade em um cenário que o usuário necessite acessar os dados médicos do seu RES, mas está em outro lugar onde o padrão utilizado é diferente. Por exemplo, um usuário possui um RES em um Plano de Saúde privado no Brasil (FHIR) e tenta acessá-lo de um hospital em um país na Europa (OpenEHR).

No SmartRES o componente MARCIA auxilia no processo de aquisição dos dados, sendo um mecanismo para acesso e coleta dos dados do componente RES, enquanto o componente GIRLS se apresenta como uma funcionalidade extra, uma vez que ele permite a interoperabilidade de padrões. Assim, os componentes MARCIA e o RES se comunicam a fim de retornar todo o histórico de saúde do usuário. Dessa forma, o componente RES do usuário poderá ser acessado de qualquer lugar, alimentando os dados devidos para processamento do componente CloudRES no Módulo Nível de Risco.

\subsection{Módulo Nível de Risco}

O componente CloudRES é responsável pelo cruzamento dos dados do componente RES do paciente e dos seus dados coletados em tempo real pelos sensores (Módulo Sensores). Dessa forma, é possível analisar qual o nível de risco desse usuário através de predição (padrões aprendidos). Assim, o Módulo Nível de Risco serve como um filtro de dados antes de se chegar ao Módulo Business Intelligence (BI), auxiliando na toma de decisão. A classificação do risco neste modulo possui quatro níveis, descritos a seguir:

- Normal: Indica situação estável. Os dados do usuário serão enviados para o BI e salvos na aplicação MobileRES, para que possam ser visualizados tanto pelos médicos, quanto pelo usuário.

- Baixo: envia alerta ao usuário que terá acesso a um chatbot, via MobileRES, para tirar dúvidas, se assim o desejar, e auxiliá-lo na tomada de decisão.

- Médio: trata-se de uma classificação configurável para cada usuário, a partir de uma análise a ser feita por uma equipe de saúde.

- Alto: Considerado situação crítica, necessitando de procedimentos imediatos: acionamento de contatos de urgência (médicos, familiares), envio de ambulância ao local, etc.

\subsection{Módulo Business Intelligence}

O Business Intelligence (BI) pode ser definido como o conjunto de métodos, processos e políticas que visam obter informações extraídas de grandes bancos de dados, com o objetivo de definir estratégias de negócios. No contexto do SmartRES, esse módulo será responsável por receber e avaliar os dados individualmente aos usuários. Esses dados chegam na central, composta por médicos e enfermeiros capacitados, para realizar a análise dos alertas. Se o alerta recebido pela central tiver sua relevância (ou seja, não ser um alerta falso), serão então iniciadas ações, comentadas no Módulo Nível de Risco, para auxiliar o usuário: chatbot, ambulância (realização do socorro), alertas para o médico e alertas para o próprio usuário e familiares.

\subsection{Módulo Serviços}

Este Módulo é constituído por três componentes: MobileRES, ChatBot, Alertas.

O componente MobileRES é a aplicação mobile responsável por armazenar todos os dados coletados pelos sensores a ela associados. Esses dados, após processados pelos demais módulos, serão então apresentados em um dashboard para serem visualizados 
pelo usuário. No momento em que um dos dados coletados sofre alguma variação substancial (caracterizando um problema de saúde), é então criado um alerta para ser enviado ao CloudRES, onde será avaliado e classificado o nível de risco da situação. Além disso, é através do MobileRES que ações como o chatbot e medidas de urgência ocorrem, ou são acionadas. Essa aplicação traz para o usuário uma facilidade no processo de monitoramento, pois ele poderá saber em tempo real como está a sua saúde. Essa facilidade, no contexto de pacientes em estado critico é fundamental, haja vista que o tempo de ação é crucial.

O componente ChatBot é uma funcionalidade dentro da aplicação MobileRES que fornece uma maior assistência ao usuário, seja em momento de risco ou não. O usuário a qualquer momento poderá acessar esse bot e tirar dúvidas referentes ao seu contexto de saúde. O Chatbot do SmartRES utiliza mecanismos de Processamento de Linguagem Natural e, futuramente, Machine Learning (ML) [Valter et al. 2019] para responder ao usuário da melhor forma, considerando seu perfil e sua resposta a variáveis dentro do contexto em que ele se insere.

O componente Alertas do Módulo Serviços funcionará como um mecanismo de notificação para médicos, familiares e para o próprio usuário. Será por meio desses alertas que ações de prevenção ou intervenção serão tomadas a fim de manter a saúde e o bem estar de quem está sendo monitorado. Dessa forma, o SmartRES cria um aparato de monitoramento e assistência ao usuário.

\section{SmartRES no contexto da COVID-19}

A plataforma SmartRES foi planejada, inicialmente, para a prevenção e reação a situações clássicas de acidentes de saúde (desmaios, quedas, mal-estar, etc). No entanto, é natural, e um desafio, imaginarmos também seu uso no combate à COVID-19, essa doença que assola a humanidade em 2020.

Nesta seção, mostramos um cenário de aplicação do SmarRES associando-o a sua arquitetura funcional, mostrada na Figura 01. Em seguida, usamos neste cenário, os níveis de alerta do SmartRES para ajudar o usuário na questão da contaminação da COVID-19.

A ideia do SmarRES é fazer o cruzamento de dados em tempo real do usuário (seus sinais vitais captados pelos sensores) com dados de seus histórico clinico (componente RES do SmarRES). Para que o SmarRES possa avaliar o nível de risco, ele faz uso do arquétipo da COVID-19 e do componente MARCIA (ver Figura 4), a exemplo do realizado em [Gomes et al. 2018] com chikungunya.

\subsection{Cenário de Aplicação do SmartRES}

Considere, no contexto da arquitetura funcional do SmartRES (Figura 1), um usuário com a pulseira, os sensores e a aplicação APP-SmartRES no seu smartphone, como ilustrado na Figura 2.

A pulseira captura os dados do usuário, via sensores, e "os envia"para a aplicação APP-SmartRES. Em uma versão preliminar, já implementadas; esses dados são tratados e analisados APP-SmartRES; quando detectada uma variação substancial (por exemplo, pressão acima do limite normalmente estabelecido), é gerado um alerta para o usuário. 
Figura 2. Cenário de Aplicação - Alto Risco.

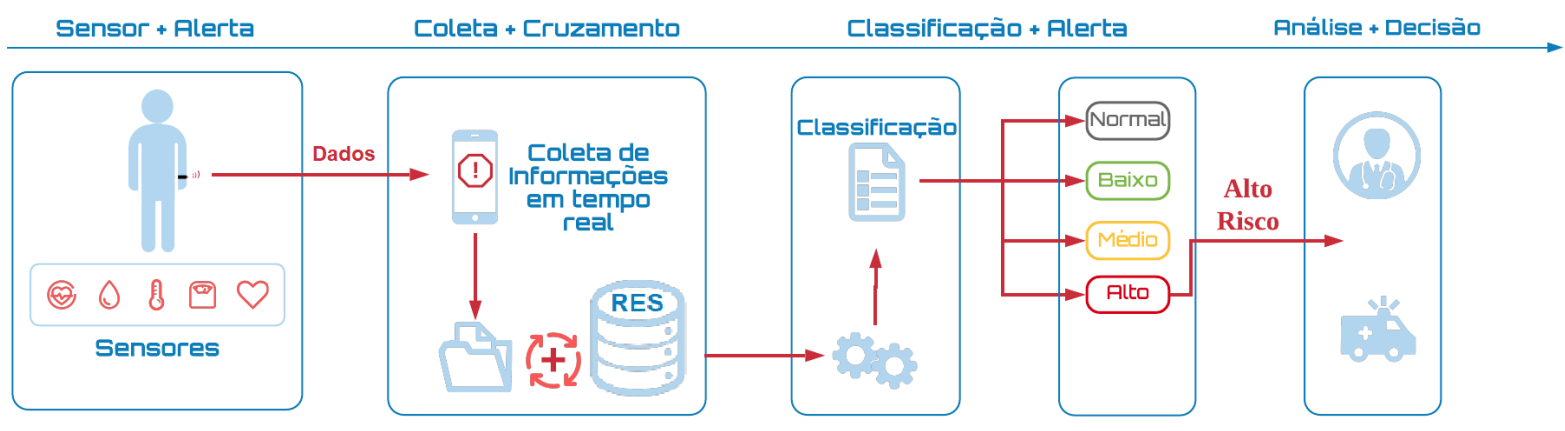

Na versão proposta pelo SmartRES neste trabalho (Figuras 1 e 2) o tratamento e análise do dados são feitos pelo CloudRES. Este componente avalia o nível de risco a partir dos dados recebidos do usuário, em tempo real (Módulo Sensores), cruzando-os com dados do usuário provenientes do Módulo de Aquisição. A partir dai, é possível ao Módulo Nível de Risco classificar o grau de risco desse usuário (o quão essa pressão alta é grave no seu contexto histórico (modelos preditivos e de inferência) de saúde daquele usuário).

Ao classificar como Alto Risco (caso ilustrado na Figura 2), o Módulo Serviço acionaria uma série de ações, desde medidas locais ao usuário (Alertas e Chatbot, via MobileRES, a medidas remotas de urgência (contato com medico e familiares, envio de ambulância, etc).

O procedimento lógico que suporta essa classificação do usuário pelo SmartRES é o mesmo utilizado em seu uso tradicional, ou seja, o cruzamento de dados em tempo real (Módulo Sensores, via componente MobileRES) com dados do histórico clínico do usuário (Módulo Aquisição de dados), usando um dos mecanismos:

1) Utilização de arquétipos existentes ou construídos com o MARCIA: tratam-se de protocolos cujos templates são criados a partir de conhecimento tácito e de heurísticas dos especialistas. de ML.

2) Utilização de modelos preditivos: este procedimento faz uso de rotinas clássicas

\subsection{Arquétipo da COVID-19 no SmartRES}

A proposta neste trabalho tem como objetivo o usuário ser ajudado pela plataforma quanto a possibilidade dele ser alertado quanto a possibilidade de estar contaminado pela COVID-19 nos níveis de alerta SmartRES: normal, médio, baixo e alto risco.

Quando há novos eventos que precisam ser registrados no sistema, a rotina tradicional é a alteração do sistema para inserir uma nova funcionalidade, implicando em mudança de código e reteste do sistema. A justificativa do sistema MARCIA na plataforma do SmartRES é que o mesmo funcionará como uma interface, recebendo e processando dados clínicos, representados por meio de arquétipos ("padrões de metadados openEHR que representam dados clínicos”) [Gomes et al. 2018]. 
A Figura 3 mostra o arquétipo da COVID-19, denominado "openEHR-Confirmed Covid-19 infection report.v0", disponibilizado no CKM (Repositório Mundial de Arquétipos).

Esse arquétipo representa as informações clínicas sobre o COVID-19, conforme as diretrizes da Organização Mundial da Saúde (OMS).

Figura 3. Mapa mental do Arquétipo COVID-19. Fonte: openehr.org/

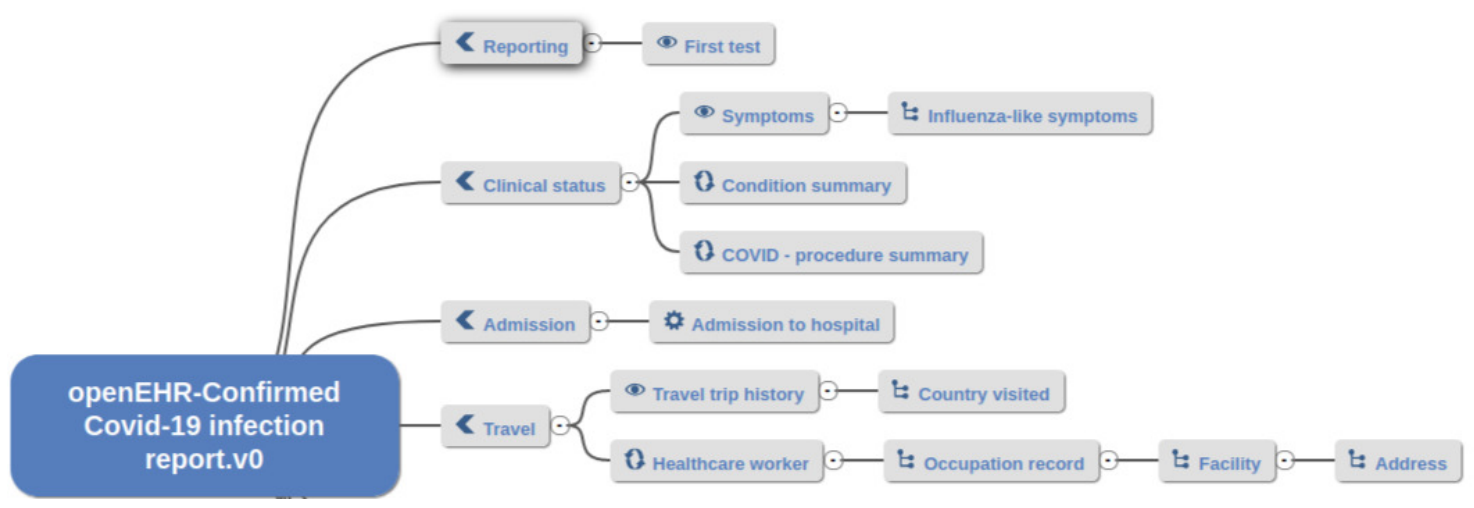

O arquétipo "openEHR-ConfirmedCovid-19 infection report.v0" possui os seguintes atributos:

- Comunicando (Reporting): comunica a informação sobre o primeiro teste (first test) realizado pelo usuário;

- Estado Clínico (Clinical status): informa os sintomas ("symptoms") que o paciente está sentindo, como: dores, falta de ar, sintomas semelhante à gripe ("influenza-like symptoms");

- Admissão (Admission): informa sobre a admissão ou interação hospitalar (admission to hospital);

- Viagem (Travel): fornece informações sobre o recente histórico de viagens do usuário (travel trip history), se o mesmo é profissional de saúde ou tem contato com alguém que trabalha na área da saúde (healthcare worker), se visitou algum País (country visited), qual foi o registro de ocupação (occupation record), onde se instalou (facility) e qual o endereço (address).

Esses dados são trabalhados no sistema MARCIA com regras heurísticas e, em seguida, são enviados para o componente RES da plataforma do SmartRES. No modelo baseado em arquétipos não é necessário alterar o código fonte para acomodar esta nova funcionalidade, atendendo rapidamente a dinâmica que exigem os incidentes da área da saúde, em especial os incidentes causados pela COVID-19.

Considerando o contexto atual da COVID-19, é natural que informações atuais sobre esta doença não façam parte do RES do paciente e que o conhecimento técnico e cientifico adquirido sobre a COVID-19, que cresce a cada momento, também não esteja disponibilizado (ainda) na plataforma. O componente MARCIA do Módulo Aquisição de Dados da plataforma SmartRES (ver Figura 1) tem essa tarefa de "consumir"novos arquétipos (ler, processar, armazenar novos dados clínicos) dinamicamente, ou seja, ele se adapta facilmente às inovações. 


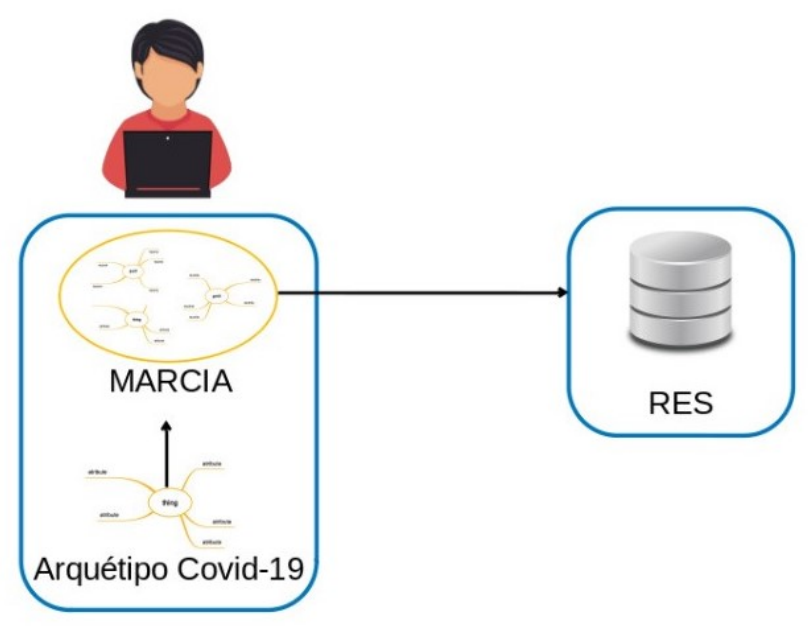

A Figura 4 mostra o MARCIA "consumindo" o arquétipo da COVID-19, tornando-o visível no componente RES da plataforma SmartRES. Assim, o MARCIA pode ler e processar o arquétipo (informação clínica) da COVID-19, já disponibilizado no CKM (Repositório Mundial de Arquétipos), que foi definido conforme o protocolo da OMS. Em seguida, o MARCIA agrega esse novo conhecimento clínico no componente RES do paciente, usuário da plataforma. Desta forma, o componente MARCIA do Módulo de Aquisição de Dados do SmartRES funciona como uma interface de atualização rápida do RES, sem mudanças no código fonte do mesmo, mas agregando uma nova funcionalidade em tempo real.

\section{Aspectos de Implementação}

O SmartRES (ver Figura 1) é resultado de diversos trabalhos realizados no Laboratório de Redes de Computadores e Sistemas (LAR), do IFCE campus Aracati. Ele tem sua origem no NextSAUDE, projeto financiado pela Fundação Cearense de Apoio ao Desenvolvimento Cientifico e Tecnológico (FUNCAP), em 2015.

Do projeto NextSAUDE nasce o VITE das Coisas [Melo 2017], um sistema sensível a contexto (contexto-aware concept) que propõe o Health OpenIoT, uma plataforma baseada na arquitetura $O p e n I o T^{4}$.

A Figura 5(a), mostra a pulseira desenvolvida no protótipo do VITE das Coisas, composta por sensores de SPO2, Temperatura, BPM, acelerômetro (detecção de quedas), utilizada no SmartRES.

A Figura 5(b), monstra a tela de visualização dos dados para o usuário do aplicativo do SmartRES (APP-SmartRES), coletados pelos sensores. No APP-SmartRES os valores de máximo e mínimo são configurados manualmente pelo usuário.

Conforme comentado na introdução deste trabalho, os componentes MARCIA e GIRLS foram implementados, faltando a integração deles com o componente RES na

\footnotetext{
${ }^{4} \mathrm{http}: / /$ www.openiot.eu
} 


\section{Figura 5. VITE das Coisas}

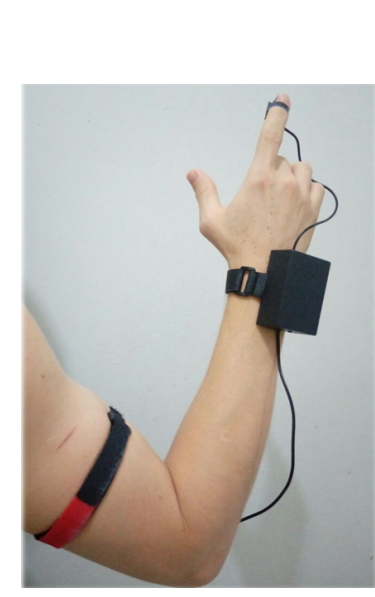

(a) Hardware do VITE das Coisas.

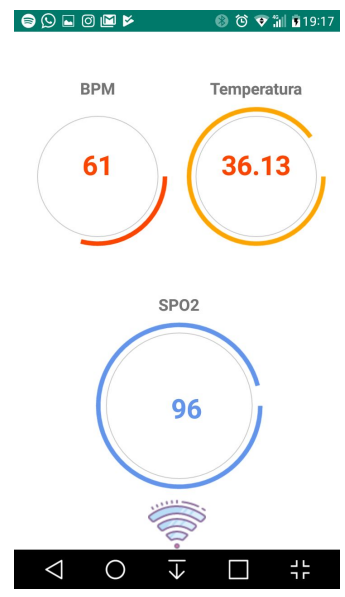

(b) Dashboard do APPSmartRES.

definição do Módulo Aquisição de dados no SmartRES. O componente ChatBot foi implementado e está sendo integrado ao SmartRES. Restam, portanto, a especificação e implementação do Módulo Nível de Risco para a completude da arquitetura funcional do SmartRES, apresentada na Figura 01.

No que diz respeito ao uso de SmartRES no contexto da COVID-19, trabalho similar foi realizado pela equipe com o arquétipo do chikungunya [de Souza 2017], resultando em importante contribuição para o Repositório Mundial de Arquétipos(CKM).

\section{Considerações Finais}

O avanço tecnológico e o envelhecimento da população brasileira tem exigido, cada vez mais, soluções eficientes no monitoramento na área de saúde. Estas soluções têm sido obviamente requisitadas por usuários com doenças crônicas, mas também por pessoas jovens e saudáveis. A pandemia causada pela COVID-19 agravou, sobremaneira, esse quadro e criou novas necessidades de prevenção.

A arquitetura SmartRES proposta neste trabalho se apresenta como uma solução inovadora de baixo custo que faz uso de IoT e sistemas inteligentes na prevenção (modelos preditivos e inferência) e/ou (re)ação de acidentes de saúde que podem acometer seu usuário. Neste trabalho ilustrou o uso do SmartRES com a COVID-19, dada as urgências que o fato impõe.

Resultado da experiência de vários trabalhos acadêmicos e prototipação na área de IoT em Saúde, Padrões de RES e da aplicação de ML em saúde, o SmartRES sinergiza os esforços de três grandes projetos desenvolvidos no LAR (Laboratório de Redes de Computadores e Sistemas) de Aracati: GIRLS (interoperabilidade), MARCIA (gerenciamento de prontuário) e VITE das Coisas (coleta e tratamento de dados em tempo real). Além da contribuição acadêmica, a expectativa operacional do SmartRES é tornar-se uma opção de baixo custo, comparado a similares disponíveis no mercado, proibitivos às camadas populares.

A expectativa dos autores deste trabalho é fazer o monitoramento inteligente de 
forma contínua, proporcionado pelo SmartRES, sendo um mecanismo eficiente para a melhoria da qualidade de vida, em momento tão peculiar da humanidade.

\section{Referências}

Atenção à Saúde, S. (2015). Conjunto mínimo de dados da atenção à saúde. In I Encontro Nacional do Conjunto Mínimo de Dados da Atenção à Saúde. SUS.

Braga, R. D. et al. (2014). Registro eletrônico em saúde: proposta de um modelo de informação para uso na atenção primária com vistas à interoperabilidade.

Costa, C. M., Menárguez-Tortosa, M., and FernáNdez-Breis, J. T. (2011). Clinical data interoperability based on archetype transformation. Journal of biomedical informatics, 44(5):869-880.

Costa, P. M. B. d. P. (2018). Sistema de internet das coisas para o apoio aos cuidados de saúde da população idosa. Master's thesis, Universidade de Évora.

de Souza, F. J. G. (2017). MARCIA, UMA METODOLOGIA PARA O MANEJO DE REGISTRO CLÍNICO COM USO DE ARQUÉTIPOS PARA INTEROPERABILIDADE EM SISTEMAS DE SAÚDE. Dissertação (Mestrado Profissional em Computação Aplicada do Programa de Pós-Graduação em Ciência da Computação do Centro de Ciências e Tecnologia), UECE (Universidade Estadual do Ceará), Fortaleza - Ceará, Brasil.

Gomes, F., Freitas, R., Ribeiro, M., Moura, C., Andrade, O., and Oliveira, M. (2019). Girls, a gateway for interoperability of electronic health record in low-cost system:* interoperability beetween fhir and openehr standards. In 2019 IEEE International Conference on E-health Networking, Application \& Services (HealthCom), pages 1-6. IEEE.

Gomes, F., Paiva, J., Bezerra, A., Moura, C., Oliveira, M., and Andrade, O. (2018). Marcia: Applied clinical record management: Eletronic health record applied with ehrserver. In 2018 IEEE 20th International Conference on e-Health Networking, Applications and Services (Healthcom), pages 1-6. IEEE.

Melo, E. R. R. (2017). VITE DAS COISAS, UMA SOLUÇÃO PARA URGÊNCIA E EMERGÊNCIA EM SISTEMAS DE SAÚDE. Dissertação (Mestrado Profissional em Computação Aplicada do Programa de Pós-Graduação em Ciência da Computação do Centro de Ciências e Tecnologia), UECE (Universidade Estadual do Ceará), Fortaleza - Ceará, Brasil.

Paz, L. F., Maran, V., Machado, A., and Weber, J. G. (2013). Mobidoctor: uma aplicação móvel para acesso ao registro eletrônico de saúde de pacientes. Revista Brasileira de Inovação Tecnológica em Saúde-ISSN: 2236-1103.

Safran, C. and Perreault, L. E. (2001). Management of information in integrated delivery networks. In Medical Informatics, pages 359-396. Springer.

Valter, R., Santiago, S., Ramos, R., Oliveira, M., Andrade, L. O. M., and de HC Barreto, I. C. (2019). Data mining and risk analysis supporting decision in brazilian public health systems. In 2019 IEEE International Conference on E-health Networking, Application \& Services (HealthCom), pages 1-6. IEEE. 